\title{
ÉDITORIAL
}

\section{Le « tout » phytothérapie}

\section{P. Goetz}

(C) Lavoisier SAS 2018

En phytothérapie, comme en médecine générale, il convient de rester «Un».

Nous avons constaté des réticences à l'énoncé de certaines vérités comme l'effet des huiles essentielles (par exemple du millepertuis), etc.

La phytothérapie est un domaine qui demande à ne pas être sectaire. Associer la médecine conventionnelle à la phytothérapie n'est pas un crime, mais relève du bon sens dans certaines conditions.

De plus en plus fréquemment, nous avons connaissance d'expérimentations permettant de vérifier que telle ou telle huile essentielle antibactérienne augmente ou complète l'effet d'un antibiotique.

Nous avons parallèlement également été informés de dérives dangereuses du commerce de plantes en Algérie qui avaient pour conséquence une absence de soins pour des personnes malades qui accordaient leur confiance à des « guérisseurs ».

C'est pourquoi il nous semble important de communiquer à la fois les bons résultats de l'usage de la phytothérapie, mais aussi les anomalies et les accidents : interaction entre racine de céleri et venlafaxine, effets psychotiques avec le fruit du Garcinia cambogia, un cas de vasoconstriction cérébrale par l'eucalyptus...

Soigner sans nuire est une constante de ladite « médecine alternative $»$.

Mais cela n'est pas évident dans la pratique quotidienne. Quand un patient se plaint d'un effet secondaire consécutif à la prise d'un phytomédicament, il convient d'accepter ce qu'il dit et d'en chercher la ou les causes, alors que rien ne le laissait prévoir. Encore faut-il que le diagnostic de la cause soit exact, que le produit délivré soit conforme à la prescription, que le médicament soit pris au bon moment, et avec quelles autres médications...

Il faut aussi considérer que tous nos patients ne répondent pas uniformément à la même médication.

C'est pourquoi l'apprentissage de la phytothérapie se fait aussi en dehors des salles de cours et dans les échanges hors séances de conférence.

Nous essayerons donc dans notre revue de donner « toutes » les informations qui permettent d'être de vrais professionnels en la matière. 